\title{
Trans-Planckian Tail in a Theory with a Cutoff
}

\author{
B. Reznik * \\ Department of Physics \\ University of British Columbia \\ Vancouver, B.C., Canada V6T1Z1
}

\begin{abstract}
Trans-planckian frequencies can be mimicked outside a black-hole horizon as a tail of an exponentially large amplitude wave that is mostly hidden behind the horizon. The present proposal requires implementing a final state condition. This condition involves only frequencies below the cutoff scale. It may be interpreted as a condition on the singularity. Despite the introduction of the cutoff, the Hawking radiation is restored for static observers. Freely falling observers see empty space outside the horizon, but are "heated" as they cross the horizon.
\end{abstract}

*e-mail: reznik@physics.ubc.ca 


\section{INTRODUCTION}

The standard derivation of the Hawking radiation [1] requires the existence of exponentially high frequency modes in a classical space-time background. Indeed after a short time, (of order $t \sim M \ln M$ ), the required frequency becomes $\omega \sim 1$ in planck units. For this reason the standard derivation can not be trusted already after few Hawking photons were emitted. It appears that in any derivation of Hawking radiation, no new physical ingredients, a naive short distance cutoff will eliminate the Hawking effect [2]. It is, of course, possible that the origin of the Hawking radiation does depend on the behavior of an ultrahigh trans-planckian spectrum. In this article however we suggest an alternative mechanism for generating Hawking's radiation in a theory with an effective cutoff.

Evidence that a theory with cutoff may reproduce the Hawking radiation has been recently provided by Unruh's work [3]. Unruh has shown that a natural cutoff still gives rise

to the Hawking radiation in the case of sonic black-holes [4]. In his approach the cutoff modifies the dispersion relation for sound waves. This, in turn, alters the motion of modes with frequency close to the cutoff scale and gives rise to a new type of trajectories which approach the horizon but eventually "reflect" back to infinity. Further works tried to adapt Unruh's model to real black-holes [5] [6] [7]. It is not clear that a similar process is indeed realized for real black-holes.

In this article we present another possibility. It is shown that even without modifying the ordinary field equations and the ensuing dispersion relations, as in the above proposals, one can still restore the Hawking radiation in a theory with a cutoff. In the present approach the Hawking radiation is generated by an apparent trans-planckian tail outside the black-hole horizon. The source of this tail is an exponentially large wave that is mostly hidden behind the black-hole horizon. To develop this picture we shall use two key ingredients:

I) Ultra-high frequency modes can be mimicked to arbitrary accuracy in a bounded region even with a finite band spectrum. 
The basic idea was discovered by Aharonov at. el. [8] and was further developed by Berry [9], who coined the term "super-oscillations" to describe such a behavior. A simple example of a function $F(t)$ which exhibits super-oscillations was given in [8]:

$$
F\left(t ; N, \omega^{*}\right)=\left[\left(\frac{1-\omega^{*} / \omega_{0}}{2}\right) e^{i t \omega_{0} / N}+\left(\frac{1+\omega^{*} / \omega_{0}}{2}\right) e^{-i t \omega_{0} / N}\right]^{N} .
$$

Here, $N>1$ is an integer, and $\omega^{*}$ and $\omega_{0}$ being the super and reference frequencies. For small $t$ we expand $\exp \left(i t \omega_{0} / N\right)$ and find:

$$
\begin{aligned}
& F\left(t ; N, \omega^{*}\right)=\left[e^{-i \omega^{*} t / N}+\frac{\left(\omega^{* 2}-\omega_{0}^{2}\right) t^{2}}{2 N^{2}}+O\left(N^{-3}\right)\right]^{N} \\
& =e^{-i \omega^{*} t}\left[1+\frac{\left(\omega^{* 2}-\omega_{0}^{2}\right) t^{2}}{2 N}+O\left(N^{-2}\right)\right] \cong e^{-i \omega^{*} t} .
\end{aligned}
$$

Although the spectrum of (11) includes only modes with frequencies $\omega \in(-1,+1)$, in the time interval $|t|<<\sqrt{N} / \sqrt{\omega^{* 2}-\omega_{0}^{2}} \equiv T, F(t)$ behaves as a wave with arbitrary large frequency $\omega^{*}$. The number of super-oscillations in this interval is $\sim \sqrt{N}$. Systems that interact with the wave $F$ only during $|t|<<T$ will not distinguish between $F$ and a pure wave $e^{-i \omega^{*} t}$ that extends for all times.

This remarkable feature is derived at the expense of having such functions grow exponentially in other regions. In the example above, for $|t|>T$, we get $F \sim e^{N}$. Nevertheless, as we shall see, the large amplitudes can be confined to a compact region. In particular, by adapting Berry's integral representation [9], the large amplitudes can be entirely confined to the interior region of a black-hole while only a high frequency tail remains outside the black-hole horizon. This "tail" will be seen by the external observer as the origin of the Hawking radiation. The observer can not probe the interior of the black-hole and can not distinguish between the mimicked tail and a "truly" trans-planckian frequency mode.

If the above function $\mathrm{F}$ is viewed as a wave function, then the probability to see a photon coming out from the tail is exponentially small. In order to avoid this, we shall make an additional assumption which is the second basic ingredient:

II) A black-hole is described by two conditions: by the ordinary ingoing state and by a final condition. 
Under this assumption, the black-hole is described in a fashion similar to that of a pre and postselected system ( [10], [11], [12]). A pre and postselected ensemble is prepared according to given initial and final conditions. Observations can be then made at some intermediate time between the pre and postselections, and the probability of the measured results can be expressed as conditional probabilities. However in our case the final state will be given a more fundamental role. It will not be determined by a postselection done by some fictitious observer in the future, rather it will be conceived as arising from some new fundamental law, which is required by the presence of a singularity in the future.

Such a final condition can be anticipated, for example, in a theory that replaces past or future curvature singularities by smooth initial or final conditions. To some extent, the Hartle-Hawking ansatz for the cosmological wave function [13] can be interpreted as corresponding to initial and final conditions. When the WKB approximation is valid, the Hartle-Hawking wave function is expressed in terms of the action $S$ as

$$
\Psi \simeq e^{i S}+e^{-i S} .
$$

It is possible to interpret these two terms as two wave functions which travel forward and backward in time, and correspond to conditions in the past or in the future. It is possible that a similar fundamental principle is available also for the case of a black-hole singularity.

Although our assumption $I I$ above might seem at first radical, we shall show, in Section 2 , that final conditions may be constructed which do not affect low energy observables. Such a final condition will manifest only in very extreme cases. The basic idea will be to implement the condition only on very high frequency modes above some scale $\omega_{h}$.

In Section 3 we shall construct the special super-oscillatory function which mimics a high frequency tail using a bounded spectrum. In Section 4 we study the response of a stationary detector in a black-hole geometry to a scalar field when a cutoff with respect to Kruskal coordinates was introduced. In section 5 the two main ingredients, namely superoscillations and a final condition, are combined for the simple case of an eternal black-hole. A cutoff is assumed with respect to the Kruskal coordinates both on the initial Kruskal vacuum 
state $\left|O_{K}\right\rangle$ and our final state $|f\rangle$. It is shown that these initial and final conditions cause the observer to see Hawking radiation emitted from the black-hole. Finally, we conclude with a discussion of our results and remaining difficulties.

\section{FINAL CONDITION ON ULTRA-HIGH MODES}

In this section it is shown that a non-trivial final condition can be imposed without affecting low energy observables. Over the last decade Prof. Y. Aharonov and collaborators have elaborated on the two vector formalism of quantum mechanics. In this formalism one specifies both an initial and a final states and considers measurements done at intermediate time. (For a detailed discussion see refs. [11] and [12].)

Let the initial and final conditions on a system be that at $t=-\infty(+\infty)$ the field is in the state $|i\rangle(|f\rangle)$. Indeed, one can in ordinary quantum mechanics impose two such conditions. These states are independent, but need to be non-orthogonal.

In the following we shall consider measurements at some intermediate time. Given an observable $A=\sum a \Pi_{a}$, where $\Pi_{a}$ are projectors to the eigenstates $|a\rangle$, the probability to measure $A=a$ is given by the conditional probability 円:

$$
\mathcal{P} \operatorname{rob}(a \mid f, i)=\frac{\mathcal{P} \operatorname{rob}(a, f \mid i)}{\mathcal{P} r o b(f \mid i)}=\frac{|\langle f \mid a\rangle\langle a \mid i\rangle|^{2}}{\sum_{a^{\prime}}\left|\left\langle f \mid a^{\prime}\right\rangle\left\langle a^{\prime} \mid i\right\rangle\right|^{2}} .
$$

For certain non-trivial final conditions, low energy laboratory experiments will not depend on the final condition. In the example considered here a final condition is imposed only on the high energy sector, i.e. only for $\omega>\omega_{h}$, where $\omega_{h}$ is some high energy scale.

To spell out this proposal, let us for simplicity consider a free massless scalar field theory in Minkowski space-time, and let us assume that in a certain rest frame the final state of the field has the form:

\footnotetext{
${ }^{1}$ Clearly (4) is different from $|\langle a \mid i\rangle|^{2}$, the probability obtained if only the initial state is fixed. The latter is obtained from (何) by further summing over $f$.
} 


$$
\left|f_{(L, F)}\right\rangle=\frac{1}{\sqrt{1+\xi^{2}}}(|L\rangle+\xi|F\rangle)
$$

where $|L\rangle$ and $|F\rangle$ are two normalized states in Fock space, and $\xi$ controls the relative probability. The first component, $|L\rangle$, denotes a low energy "laboratory state", which contains only particles of low frequency:

$$
|L\rangle=\left(1+\sum_{\omega_{k}<\omega_{h}} C_{k}(L) a_{\omega_{k}}^{\dagger}+\sum_{\omega_{k}, \omega_{l}<\omega_{h}} D_{k l}(L) a_{\omega_{k}}^{\dagger} a_{\omega_{l}}^{\dagger}+\ldots\right)\left|0_{M}\right\rangle
$$

The second term, $|F\rangle$, denotes a certain state of particles with frequencies above $\omega_{h}$ :

$$
|F\rangle=\left(\sum_{\omega_{k}>\omega_{h}} C_{k}(F) a_{\omega_{k}}^{\dagger}+\sum_{\omega_{k}, \omega_{l}<\omega_{h}} D_{k l}(F) a_{\omega_{k}}^{\dagger} a_{\omega_{l}}^{\dagger}+\ldots\right)\left|0_{M}\right\rangle
$$

We shall demand that the final state always has the form given in eq. (5), and is constrained always to includes the same high energy state $|F\rangle$. We shall not constrain the content of the low energy state $|L\rangle$. (In terms of the pre and postselection terminology, this corresponds to postselection of an ensemble with fixed $F$ but arbitrary $L$ in the specific combination of eq. (5) above.)

Since in the final condition the low energy state $|L\rangle$ is left unspecified, we need to modify eq. (田) accordingly. The probability to find $A=a$ is now given by further summing over a basis of the subspace, $\left.\mathcal{H}_{L}=\{|L\rangle\rangle\right\}$, of low energy states :

$$
\mathcal{P} \operatorname{rob}(a \mid F, i)=\frac{\sum_{L} P(a, L, F \mid i)}{\sum_{L, a^{\prime}} P\left(a^{\prime}, L, F \mid i\right)} .
$$

Thus:

$$
\operatorname{Prob}(a \mid F, i)=\frac{\sum_{L}\left|\left\langle f_{(L, F)}\left|\Pi_{a}\right| i\right\rangle\right|^{2}}{\sum_{L, a^{\prime}}\left|\left\langle f_{(L, F)}\left|\Pi_{a^{\prime}}\right| i\right\rangle\right|^{2}}
$$

We call $\Pi_{a}$ a low energy "laboratory" projector if

$$
\xi||\left\langle L\left|\Pi_{a}\right| F\right\rangle \|<\epsilon, \quad \forall L \in \mathcal{H}_{L}
$$

where $\epsilon$ is some small number. If (10) is satisfied for every eigenvalue of the operator $A$, then $A$ will be termed a low energy laboratory observable. If the initial state is taken to be 
one of the low energy states, i.e. $i \in \mathcal{H}_{L}$, then For a low energy observable eq. (9) reduces to

$$
\mathcal{P} \operatorname{rob}(a \mid F, i)=\frac{\sum_{L}\left|\left\langle L\left|\Pi_{a}\right| i\right\rangle+O(\epsilon)\right|^{2}}{\sum_{L, a^{\prime}}\left|\left\langle L\left|\Pi_{a}^{\prime}\right| i\right\rangle+O(\epsilon)\right|^{2}}=\frac{\left\langle i\left|\Pi_{a}\right| i\right\rangle}{\langle i \mid i\rangle}+O(\epsilon),
$$

the ordinary $\mathrm{F}$ independent expression.

Nevertheless, if the initial state does contains states with $\omega>\omega_{h}$, or when the condition (10) is not satisfied, the full expression (9) must be used, and the probability generally depends on $|F\rangle$.

Although we have seen that expectation values for low energy laboratory observables reduce to the ordinary expression, it is possible that the fluctuations of the field are still sensitive to the condition $F$. To investigate this question let us consider the case of continuous measurements at intermediate times. In particular let us consider the interaction of a particle detector with the field. This example will be useful in the following sections as well.

A particle detector [14] [15] can be described as a two level system with an energy gap $\Omega$. The detector is coupled to a scalar field $\phi(x, t)$ via the action:

$$
S_{I}=\lambda \int d \tau d x\left(A+A^{\dagger}\right) \phi(x, t) \delta\left(x-X_{D}\right)
$$

Here, $\tau$ is the proper time in the rest frame of the detector, $X_{D}(t)$ is the classical trajectory of the detector. $A, A^{\dagger}$ act on the two internal states $| \pm\rangle$ according to:

$$
\begin{aligned}
& A^{\dagger}|-\rangle=|+\rangle, \quad A|+\rangle=|-\rangle, \\
& A^{\dagger}|+\rangle=0, \quad A|-\rangle=0 .
\end{aligned}
$$

A detection of a particle will be described as a transition from the ground state to to the excited state. In the limit of small coupling constant, we shall be interested in obtaining the transition amplitude, computed to the first order in $\lambda$.

With the final condition, the transition probability is given by:

$$
T(+\mid-, F, i)=\frac{\sum_{L}\left|\left\langle+, f_{(L, F)}\left|U_{I}\right|-, i\right\rangle\right|^{2}}{\sum_{ \pm, L}\left|\left\langle \pm, f_{(L, F)}\left|U_{I}\right|-, i\right\rangle\right|^{2}}
$$


where $\sum_{ \pm}$denotes a summation over the final internal states, and $U_{I}=\exp \left(-i \int L_{I} d \tau\right)$ is the unitary evolution operator in the interaction picture.

To lowest order in the coupling constant we get:

$$
\begin{gathered}
T(+\mid-, F, i)=\lambda^{2} \frac{\sum_{L}\left|\left\langle+, f_{(L, F)}\left|\int L_{I} d \tau\right|-, i\right\rangle\right|^{2}}{\sum_{L}\left(\left|\left\langle f_{(L, F)} \mid i\right\rangle\right|^{2}+\lambda^{2}\left|\left\langle+, f_{(L, F)}\left|\int L_{I} d \tau\right|-, i\right\rangle\right|^{2}\right)} \\
=\lambda^{2} \sum_{L} \mid\langle+| \otimes\left(\left.\left\langle L|+\xi\langle F|) \int L_{I} d \tau \mid-, i\right\rangle\right|^{2}+O\left(\lambda^{3}\right),\right.
\end{gathered}
$$

where in passing to the last line we used the identity $\langle F \mid i\rangle=0$.

The transition probability obtained in (15) has the ordinary form, except that now it contains the additional component

$$
A_{F}=\xi \int d \tau\left\langle+, F\left|L_{I}\right|-, i\right\rangle
$$

When $A_{F}$ vanishes eq. (15) reduces to the ordinary transition probability.

Let us now consider the new amplitude $A_{F}$. Using the representation (7) for $|F\rangle$, and neglecting possible multi-particle contributions we obtain

$$
A_{F}=\xi \sum_{k} \frac{C_{k}^{*}(F)}{\sqrt{4 \pi \omega_{k}}} \int_{-\tau_{0}}^{\tau_{0}} d \tau \exp (i \Omega \tau) \exp \left(i \omega_{k}\left(t(\tau)-k X_{D}(\tau)\right)=\sum_{\omega_{k}} A_{F}(k) .\right.
$$

For an inertial detector, $t=\tau / \sqrt{1-V_{D}^{2}}$, and we find that

$$
A_{F}(k)=\xi \frac{C_{k}^{*}(F)}{\sqrt{4 \pi \omega_{k}}} \frac{\sin \left[\left(\Omega+\left(\omega_{k}-C_{D} k\right) / \sqrt{1-V_{D}^{2}}\right) \tau_{0}\right]}{\left(\Omega+\left(\omega_{n}-V_{D} k\right) / \sqrt{1-V_{D}^{2}}\right) \tau_{0}}
$$

The last eq. reduces to $\delta\left(\Omega+\left(\omega_{n}-V_{D} k\right) / \sqrt{1-V_{D}^{2}}\right)=0$ only when $\tau_{0}>>1 / \omega_{k}>1 / \omega_{h}$, and provided that $\xi$ is finite, say $\xi \sim 1$. This means that as long as the relative amplitude $\xi$ of $|F\rangle$ is not large, the fluctuations are averaged out to zero after a time which is determined by $1 / \omega_{h}$. Intuitively this seems natural. An interaction on a time scale shorter then $1 / \omega_{h}$ involves energy fluctuations of order $\sim \omega_{h}$, which in turn depend on the condition $F$.

Anticipating the discussion in Section 5, let us also consider the large $\xi$ case. By insisting that eq. (10) is satisfied we find that the expectation value (11) are still unmodified. 
Nevertheless, the fluctuating $A_{F}$ seen by a particle detector are not negligible. In this case, to average out such fluctuations we will need times $\tau_{0}>>/ \omega_{h}$. Otherwise our detector will observe particles which are not present in the initial state $|i\rangle$.

Finally we note that the above considerations can be easily extended to the case of a final mixed state. The analog of the state $\left|f_{(L, F)}\right\rangle$ is given by the density matrix

$$
\rho_{f}=|L\rangle\langle L|+\rho_{F},
$$

where $\rho_{F_{c}}$ is constructed from states with frequency $\omega>\omega_{h}$.

\section{ULTRA-HIGH FREQUENCY FROM A BOUNDED SPECTRUM}

The other key element I of our approach is the use of super-oscillatory functions, alluded to in the introduction. These functions, having only a bounded Fourier spectrum, can still mimic an arbitrarily high frequency though in a finite region.

In the Fourier representation of such a function

$$
\Phi(u)=\int_{0}^{1} d \omega C_{\omega} \exp i \omega u
$$

the trick is to choose certain coefficients $C_{\omega}$, such that at a finite interval of $u, \Phi$ exhibits rapid oscillations with a frequency $\omega^{*}>>1$.

As super oscillations necessitate large amplitudes at other regions, our purpose is to find a representation in which these large oscillations are confined to a bounded region of $u$. To construct such a function we will use a variant of an integral representation for a super-oscillatory function that was found by Berry [9]. Consider the function:

$$
\Phi_{A, \Delta}(u)=\frac{1}{\sqrt{2 \pi \Delta^{2}}} \int_{0}^{2 \pi} d \alpha e^{\frac{i}{\Delta^{2}} \cos (\alpha-i A)} e^{i \cos \alpha u}
$$

where $A$ and $\Delta$ are real parameters. The modes of $\Phi_{A, \Delta}(u)$ are bounded by $|\omega|=|\cos \alpha|<1$.

The integral above can be analytically performed to yield

$$
\Phi_{A, \Delta}(u)=\sqrt{2 \pi / \Delta^{2}} I_{0}\left(\frac{1}{\Delta^{2}} \sqrt{1+2 \cosh (A) \Delta^{2} u+\Delta^{4} u^{2}}\right)
$$


where $I_{0}$ is the zeroth modified Bessel function.

Expanding (22) around $u=0$, we note that $\Phi_{A, \Delta}(u)$ behaves as

$$
\Phi_{A, \Delta}(u)=\exp (i \cosh (A) u)
$$

$\Phi_{A, \Delta}(u)$ "super-oscillates" with frequency $\omega^{*}=\cosh A$. This expansion is valid in a region $|u|<\left(\cosh (A) \Delta^{2}\right)^{-1} \equiv \delta u$. Thus the parameter $\Delta$ controls the number, $n_{S}$, of superoscillations: $n_{S} \sim \delta u / \omega^{*}=1 / \Delta^{2}$.

By modifying the two parameters $A$ and $\Delta$, we can control the frequency and number of super oscillations. However, the limits $A \rightarrow \infty$ or $\Delta \rightarrow 0$ are singular. Outside the region $\delta u$ where the function super-oscillates, $\Phi$ grows exponentially. $\Phi$ gets its maximal value at $u=-\cosh A / \Delta^{2}$, where the amplitude grows to

$$
\Phi \sim \exp \left(\cosh A / \Delta^{2}\right)
$$

The super-oscillations are hence found at the tails of an exponentially high peak.

Far away from the region of super-oscillations, for $u>>\delta u$, (22) reduces to a low frequency wave:

$$
\Phi_{A, \Delta}(u)=\frac{1}{u} \exp (i u)
$$

In Section 5 we will show that these properties of $\Phi$, allow finding a state $|F\rangle$ that mimics the trans-planckian Hawking photons close to the horizon. A particle detector will not distinguish between a "fake" tail of super-oscillations and "real" trans-planckian model. Before proceeding to this final task, it will be useful to re-examine the interaction of a particle detector with a scalar field near a black-hole.

\section{PARTICLE DETECTOR IN KRUSKAL GEOMETRY WITH A CUTOFF}

In this section we examine the response of a particle detector in the space-time of an

eternal black-hole. We shall assume that the initial state is the Unruh vacuum [14], but 
that modes above a certain frequency are cutoff. In this section we use usual, only preselected, quantum mechanics framework. (We shall defer the discussion of an additional final condition to the next section.)

The geometry of an eternal black-hole is described in terms of Kruskal coordinates $U, V$, that are defined via the relations:

$$
\begin{gathered}
d s^{2}=(1-2 M / r) d t^{2}-(1-2 M / r)^{-1} d r^{2}-r^{2} d \Omega^{2} \\
=(1-2 M / r) d u d v-r^{2} d \Omega^{2} \\
=\frac{\exp (-r / 4 M)}{r / 2 M} d U d V-r^{2} d \Omega^{2}
\end{gathered}
$$

Here,

$$
u, v=t \pm r^{*}
$$

where $r^{*}=r+2 M \ln (r / 2 M-1)$, and

$$
U=-4 M \exp (-u / 4 M), \quad V=4 M \exp (v / 4 M)
$$

The Unruh vacuum corresponds to an initial state which reproduces Hawking radiation at $\mathcal{I}^{+}$. It is imposed by selecting the in-vacuum with respect to the Killing vector $\partial_{U}$ on the past horizon $(V=0)$ as follows. Consider a scalar field in the reduced $1+1$ spherical approximation. By the conformal invariance we have

$$
\phi(U, V)=\phi_{R}(U)+\phi_{L}(V) .
$$

In terms of creation and annihilation operators we have

$$
\phi_{R}(U)=\int \frac{d \omega}{\sqrt{4 \pi \omega}}\left(e^{-i \omega U} a_{\omega}^{R}+\text { h.c. }\right)
$$

and a similar expression of the left moving part $\phi_{L}$.

Now for the Unruh vacuum $\left|0_{U}\right\rangle$

$$
a_{\omega}^{R}\left|0_{U}\right\rangle=0
$$


Here we furthermore assume that modes with $\omega>\omega_{c}$ are cutoff.

Let us consider now a static particle detector that is located at a constant radius $r$ and interacts with the cutoff vacuum state defined above. The trajectory $(r, t)$ of the detector, can be described in terms of the Kruskal coordinates $(U, V)$ as

$$
U_{D}=-(r / 2 M)^{1 / 2} l \exp [(-t+r) / 4 M], \quad V_{D}=(r / 2 M)^{1 / 2} l \exp [(-t-r) / 4 M],
$$

where $l \equiv 4 M(1-2 M / r)^{1 / 2}$.

Using the interaction (12), and eqs. (30,32), we shall obtain the transition amplitude from an initial vacuum state an unexcited detector to a final state with excited detector state and a one scalar photon of frequency $\omega$ :

$$
A\left(+, \omega \mid-, 0_{K}\right)=\lambda \int d \tau\left\langle+, 1_{\omega}\left|\left(A+A^{\dagger}\right) \phi\left(U_{D}\right)\right|-, 0_{U}\right\rangle
$$

where the time coordinate is related to the proper time by $d t=d \tau /(1-2 M / r)^{1 / 2}$. We find that:

$$
A\left(+, \omega \mid-, 0_{U}\right)=\lambda^{2} \int \frac{d \tau}{\sqrt{4 \pi \omega_{k}}} e^{i \Omega \tau} \exp \left(i \omega_{k}(r / 2 M)^{1 / 2} l \exp [(-t(\tau)+r) / 4 M]\right)
$$

The total probability of jumping to an exited state is obtained by summation over the final emitted photons states:

$$
\mathcal{P r o b}(+)=\sum_{\omega<\omega_{c}}|A(+, \omega)|^{2}
$$

By inspecting the transition amplitude eq. (34), one finds that the integral is dominated by a stationary point at

$$
\omega_{s . p}=-4 M \exp [(t(\tau)-r) / 4 M]\left(\frac{2 M}{r}\right)^{1 / 2}
$$

Since the maximal frequency is $\omega_{c}$, after u-time of order $t-r \sim 4 M \ln \left(\omega_{C} / 4 M\right)$ this transition amplitude vanishes. In other words, the emission seen by the detector will come to halt very shortly after it started. This corresponds to the usual result that a cutoff will terminate the Hawking radiation. 


\section{RESTORING THE HAWKING RADIATION}

We shall now show that a particular choice for the final condition, gives rise to an effective ultra-high frequency in the vicinity of the horizon and avoids the above "extinction" of the Hawking radiation due to the cutoff.

As we have seen in Section 2, when a final condition on the high frequency modes is imposed, the transition amplitude (15) contains an extra term (16). The contribution of this term to the transition amplitude is

$$
A_{F}=\lambda \int d \tau\left\langle+, F\left|L_{I}\right|-, 0_{U}\right\rangle
$$

In order to replace the contribution of a trans-planckian frequency $\omega^{*}$ by a superoscillation, we shall require that

$$
\left\langle+, F_{\omega^{*}, \Delta}\left|L_{I}\right|-, 0_{U}\right\rangle=\left\langle+, \omega=\omega^{*}\left|L_{I}\right|-, 0_{U}\right\rangle
$$

We shall assume that the final state contains only modes with frequencies $\omega \in\left(\omega_{c}, \omega_{c}-\zeta\right)$, with $\omega$ in $M_{P L}$ units. $\zeta<1$ is some pure number that defines the size of the high energy "band" below the cutoff scale. Using eqs. (7.21), together with the constraint (38), we can find the coefficients $C_{k}(F)$ of the single particle states in (7). (In this article we shall not construct the coefficients of multi-particle terms.) The result is:

$$
\left|F_{\omega^{*}}\right\rangle=\int_{0}^{2 \pi} d \alpha \sqrt{\frac{\omega_{\alpha}}{\omega^{*}}}\left[\exp \frac{i}{\Delta} \cos (\alpha-i A)\right] a_{\omega_{\alpha}}^{\dagger}\left|0_{U}\right\rangle
$$

where

$$
\omega_{\alpha}=\omega_{c}+\zeta(\cos \alpha+1) / 2, \quad \omega^{*}=\cosh A .
$$

Outside the black-hole horizon, the effective transition amplitude (37), (with a sufficiently large number of super-oscillations $n_{S} \sim 1 / \Delta$ ), is precisely identical to that obtained without the cutoff. Therefore, we have shown that by a superposition of final states with $\omega \in \omega_{c} \pm 1$, we can mimic a trans-planckian frequency $\omega^{*}=\cosh A>>\omega_{c}$. 
Freely falling detectors are equivalent to inertial detectors in Minkowski space-time. We have seen in Section 2, that inertial detectors with a small boost factor, will not respond to the final condition. In close analogy, freely falling detectors outside the black-hole respond only to low frequencies modes. Thus, a freely falling detector outside the black-hole effectively interact only with a normal wave and hence sees the space as mostly empty; precisely as in the ordinary picture. Nevertheless, the standard picture fails at the interior of the black-hole. Inside the black-hole the effective trans-planckian tail rises sharply to a tremendous amplitude (24) of $\sim \exp \left(\omega^{*} / \Delta^{2}\right)$.

Although the expectation values of low-energy observable may remain unchanged, the fluctuations become exponentially large. This implies that a probe that couples during a finite time to the field will detect particles with high probability. In the standard picture the black-hole is basically empty, and nothing extrordinary occurs when an observer crosses the horizon. In our case, we expect that as the probe crosses the horizon it will be immediately start heating up! We see that in this scenario, while outside the black-hole the ordinary predictions are respected, new physics is predicted for the region hidden by the horizon.

So far we have only demonstrated that a single trans-planckian mode can be restored. To restore the full transition probability (35) we need to mimic the full trans-planckian spectrum. Since the different frequencies superpose with no interference, we need to chose a final state which is a density matrix:

$$
\rho_{f}=|L\rangle\left\langle L\left|+\int_{0}^{\omega_{\max }} d \omega^{*}\right| F_{\omega^{*}}\right\rangle\left\langle F_{\omega^{*}}\right|
$$

The first term represents the non conditioned final low energy state, which allows to restores ordinary low energy physics outside the black hole. The second term represents the final condition on high energy states near the cutoff $\omega_{c}$ and is responsible for the Hawking radiation. Strictly speaking, the state $\left|F_{\omega^{*}}\right\rangle$ become ill defined in the limit $\omega^{*} \rightarrow \infty$. Yet during every finite life time we have a well defined expression. For example, for an isolated black hole that evaporates according the standard picture during $t \sim M^{3}$, we will require in (41) $\omega_{\max } \sim \exp M^{2}$ 


\section{DISCUSSION}

In this article we have presented a novel picture in which the Hawking radiation can be restored without trans-planckian modes. The source of the radiation in this picture is a tail of trans-planckian oscillations near the horizon, that reaches out from a wave with exponentially high amplitudes hidden inside the black-hole. Consequently, the interior of the black-hole becomes "hot" for a freely falling observer that crosses the horizon. We therefore expect large back-reaction effects that drastically modify the interior geometry.

Another new aspect of our approach is the final condition on the high energy sector. An appealing motivation for this new physical condition is the existence of a future singularity. The condition may be a result of a new physical principle that replaces the future singularity by a final state $|F\rangle$.

The final state of the high energy sector near the cutoff energy seems to be a density matrix. A possible explanation for this could be the following. In our approach gravity is essentially treated semi-classically. If indeed the final state is related to the singularity, we can not expected it to be a expressed as direct product of a matter states and a semi-classical gravity state. Rather it should be a highly entangled matter-gravity state. Since outside the black-hole the semi-classical approximation is valid, the matter state should result from tracing over gravity states. This procedure may lead to a reduced density matrix like in eq. (41).

The mechanism presented in this paper is far from been complete, and many important questions remain. For example, can F states also mimic the two-point correlation functions? Or how does the radiation energy transfer in this picture? We hope that some of the features presented in this approach will turn out useful in understanding of the enigma of Hawking radiation. 
Acknoledgment I am grateful to W. G. Unruh for many helpful discussions during the preparation of this work. I also like to thank Y. Aharonov, S. Massar and S. Nussinov. for helpful discussions and remarks.

\section{Note Added}

After the completion of this work I found out that a concept related to ingredient $I$ was suggested also by H. Rosu [16]. 


\section{REFERENCES}

[1] S. Hawking, Nature, 248, 30 (1974), Comm. Math. Phys. 43, 199 (1975).

[2] T. Jackobson, Phys. Rev. D44, 1731, (1991) ibid, D48, 728 (1993).

[3] W. G. Unruh, Phys. Rev., D51, 2827 (1995).

[4] W. G. Unruh, Phys. Rev. Lett., 46, 1351 (1981).

[5] R. Brout, S. Massar, R. Parentani and Ph. Spindel, Phys. Rev. D52, 4559 (1995).

[6] T. Jacobson, Phys. Rev. D53, 7082 (1996).

[7] S. Corley and T. Jacobson, to be published in Phys. Rev. D.

[8] Y. Aharonov, D. Z. Albert, and L. Vaidman, Phys. Rev. Lett., 14, 1351 (1988).

Y. Aharonov, S. Popescu, and D. Rohrlich, "How a soft photon can emit a hard photon", Tel-Aviv preprint TAUP-1847-90, 1991.

[9] M. V. Berry, J. Phys. A: Math. Gen., 27, L391 (1994).

[10] Y. Aharonov, P. G. Bergmann and J. L. Lebowitz, Phys. Rev. B134 1410 (1964).

[11] Y. Aharonov and L. Vaidman, J. Phys. A24, 2315 (1991).

[12] B. Reznik and Y. Aharonov Phys. Rev. A52, 2538 (1995).

[13] J. B. Hartle and S. W. Hawking, Phys. Rev. D28, 2960 (1983).

[14] W. G. Unruh, Phys. Rev. D14, 870 (1976).

[15] B. S. DeWitt, in General Relativity, eds. , S. W. Hawking and W. Israel (Cambridge: Cambridge University Press), p 693, (1979).

[16] H. Rosu, superoscillations and transplanckian frequencies, gr-qc/9606070. 